\title{
Viscous torque on a sphere under arbitrary rotation
}

U. Lei ${ }^{\mathrm{a})}$

Institute of Applied Mechanics, National Taiwan University, Taipei 10764, Taiwan, Republic of China

C. Y. Yang

Department of Automatic Control Engineering, Chungchou Institute of Technology, Yuan Lin, Changhua 51003, Taiwan, Republic of China

\author{
K. C. Wu \\ Institute of Applied Mechanics, National Taiwan University, Taipei 10764, Taiwan, Republic of China
}

(Received 24 June 2006; accepted 20 September 2006; published online 30 October 2006)

\begin{abstract}
The viscous torque on a sphere was derived when the sphere is rotating with an arbitrary time varying angular velocity about a given diameter under the low Reynolds number assumption. The torque is composed of a quasisteady torque and an acceleration torque, which is an integral expression depending on the acceleration history. The acceleration torque can be neglected when the acceleration time scale is sufficiently greater than the viscous time scale, which is appropriate for particles with micron size subject to electrokinetic manipulation. () 2006 American Institute of Physics. [DOI: 10.1063/1.2372704]
\end{abstract}

Electrokinetics, including dielectrophoresis and electrorotation, are effective tools in the manipulation and characterization of particles and cells (see Refs. 1 and 2). The particles can be set into rotary motion in a rotating electric field; they can also spin as they move under the action of traveling wave dielectrophoresis generated by array(s) of electrodes as discussed by Huang et al. ${ }^{3}$ As a particle spins, it experiences a resistive viscous torque from the surrounding fluid. The viscous torque on a steady rotating sphere with radius $R$ in a still fluid with viscosity $\eta$ according to Lamb ${ }^{4}$ is

$$
\mathbf{T}=-8 \pi \eta R^{3} \boldsymbol{\Omega}_{0},
$$

where $\boldsymbol{\Omega}_{0}$ is the constant angular velocity of the sphere about a given diameter. However, both the linear and angular velocities of the particle vary as it moves since it experiences different local electric fields and/or flow fields during particle manipulation. In current electrokinetic applications, the viscous drag and torque on the sphere are evaluated using Stokes drag law and Eq. (1), respectively, with the steady linear and angular velocities replaced by their instantaneous values. It has not been examined rigorously whether such a quasisteady approximation is adequate for studying the electrokinetic manipulation.

The viscous drag on a sphere moving with an arbitrary velocity was studied extensively in literatures and a detailed result can be found from Ref. 5 (which contains also a brief literature survey on the topic). On the other hand, the viscous torque on a sphere under arbitrary rotation is not available in literatures and is the goal of the present study.

The method of analysis is similar to that for deriving the drag on a sphere moving in a still fluid with arbitrary velocity along a straight course (Ref. 6, p. 96). First we need the viscous torque on a sphere executing rotary oscillations with angular speed $\Omega=\Omega_{a} e^{-i \omega t}$, where $\Omega_{a}$ and $\omega$ are the amplitude and frequency of the oscillation, respectively. The magnitude of the torque under the low Reynolds number assumption is ${ }^{6}$

\footnotetext{
a) Author to whom correspondence should be addressed; electronic mail:
} leiu@spring.iam.ntu.edu.tw

$$
T=-8 \pi \eta R^{3} \Omega-\frac{8 \pi \eta R^{4}(1+i)}{3 \sqrt{2} \sqrt{\nu}} \frac{(-i \omega) \Omega}{(\sqrt{\omega}+(1+i) \sqrt{\nu / 2} / R)}
$$

after some algebraic manipulation, where $\nu$ is the kinematic viscosity of the fluid and $i=\sqrt{-1}$. The first term on the right hand side of Eq. (2) is the quasisteady torque. The second term involves the angular acceleration of the sphere, $-i \omega \Omega$, and is thus a term representing the effect of angular acceleration. When the sphere is rotating with an arbitrary time varying angular speed, $\Omega(t)$, about a given diameter, we can express such angular speed in terms of Fourier integral as

$$
\Omega(t)=\int_{-\infty}^{\infty} \Omega_{\omega} e^{-i \omega t} d \omega
$$

Each Fourier component, $\Omega_{\omega} e^{-i \omega t}$, contributes a corresponding torque with its magnitude

$$
\begin{aligned}
T_{\text {comp }}= & -8 \pi \eta R^{3} \Omega_{\omega} e^{-i \omega t} \\
& -\frac{8 \pi \eta R^{4}(1+i)}{3 \sqrt{2} \sqrt{\nu}} \frac{(-i \omega) \Omega_{\omega} e^{-i \omega t}}{(\sqrt{\omega}+(1+i) \sqrt{\nu / 2} / R)}
\end{aligned}
$$

according to Eq. (2). The total magnitude of the torque on the sphere can thus be calculated via

$$
T(t)=\int_{-\infty}^{\infty} T_{\text {comp }} d \omega
$$

which leads to

$$
\begin{aligned}
T(t)= & -8 \pi \eta R^{3} \Omega \\
& -\frac{4 \eta R^{4}(1+i)}{3 \sqrt{2} \sqrt{\nu}} \int_{-\infty}^{\infty} \frac{\left[\int_{-\infty}^{\infty}(d \Omega / d \tau) e^{i \omega \tau} d \tau\right] e^{-i \omega t}}{\sqrt{\omega}+(\sqrt{\nu / 2} / R)(1+i)} d \omega,
\end{aligned}
$$


TABLE I. Dimensionless torque, $T /\left(-8 \pi \eta R^{3} \Omega_{0}\right)$, at $t=t_{0}$ of the triangular forcing problem.

\begin{tabular}{rcc}
\hline \hline $\bar{t}_{0}$ & Result using Eq. (9) & Result using the superposition of Eq. (2) \\
\hline 1 & 1.1908 & 1.1704 \\
10 & 1.0276 & 1.0265 \\
100 & 1.0003 & 1.0031 \\
\hline \hline
\end{tabular}

where Eq. (3) and

$$
-i \omega \Omega_{\omega}=\frac{1}{2 \pi} \int_{-\infty}^{\infty}(d \Omega / d \tau) e^{i \omega \tau} d \tau
$$

have been employed. Equation (6) can be rearranged as

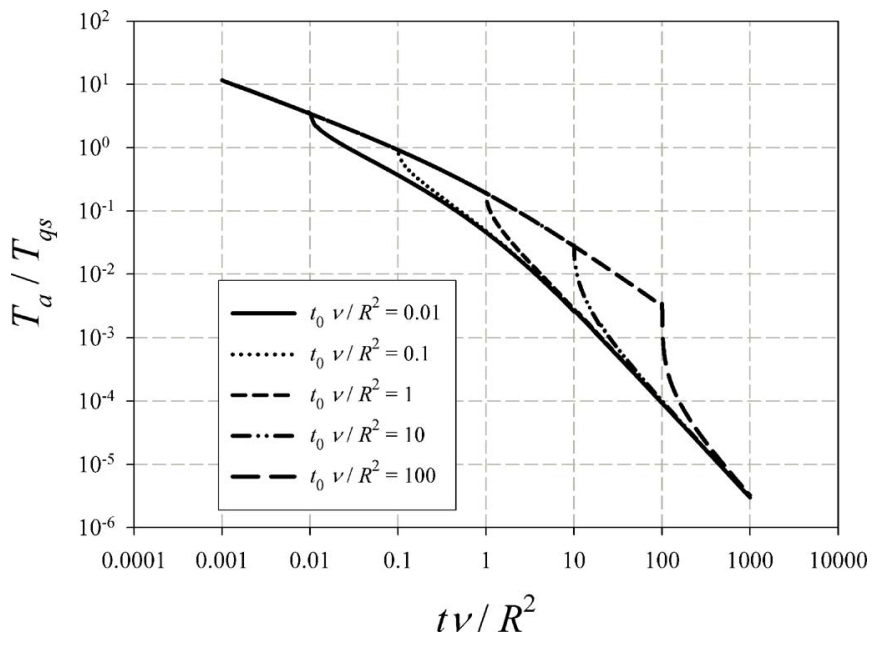

FIG. 1. Variation of the ratio of the acceleration torque to the quasisteady torque, $T_{a} / T_{\mathrm{qs}}$, with the dimensionless time normalized by the viscous time scale $\left(R^{2} / \nu\right)$ for the start-up problem.

$$
\begin{aligned}
T(t)= & -8 \pi \eta R^{3} \Omega-\frac{4 \eta R^{4}(1+i)}{3 \sqrt{2} \sqrt{\nu}} \int_{-\infty}^{t} \frac{d \Omega}{d \tau}\left\{\int_{-\infty}^{\infty} \frac{\exp [-i \omega(t-\tau)]}{\sqrt{\omega}+(1+i) \sqrt{\nu / 2} / R} d \omega\right\} d \tau \\
& -\frac{4 \eta R^{4}(1+i)}{3 \sqrt{2} \sqrt{\nu}} \int_{t}^{\infty} \frac{d \Omega}{d \tau}\left\{\int_{-\infty}^{\infty} \frac{\exp [i \omega(\tau-t)]}{\sqrt{\omega}+(1+i) \sqrt{\nu / 2} / R} d \omega\right\} d \tau .
\end{aligned}
$$

After a certain amount of manipulation, the inner integral of the second term on the right hand side of Eq. (7) is

$$
\begin{aligned}
I_{1}= & \sqrt{\frac{2 \pi}{(t-\tau)}}(1-i) \\
& -\frac{\sqrt{2 \nu}}{R}(1-i) \pi \exp \left(\frac{\nu(t-\tau)}{R^{2}}\right) \operatorname{erfc}\left(\sqrt{\frac{\nu(t-\tau)}{R^{2}}}\right),
\end{aligned}
$$

and the inner integral of the last term in Eq. (7) is identically zero. The final result according to (7) is thus

$$
\begin{aligned}
T(t)= & -8 \pi \eta R^{3} \Omega-\frac{8 \pi \eta R^{3}}{3 \sqrt{\pi}} \int_{-\infty}^{t}\left[\frac{R}{\sqrt{\nu(t-\tau)}}\right. \\
& \left.-\sqrt{\pi} \exp \left(\frac{\nu(t-\tau)}{R^{2}}\right) \operatorname{erfc}\left(\frac{\sqrt{\nu(t-\tau)}}{R}\right)\right] \frac{d \Omega}{d \tau} d \tau .
\end{aligned}
$$

As in Eq. (2), the first term on the right hand side of Eq. (9) is a quasisteady torque. The second term indicates that there is an additional viscous torque on the sphere associated with the history of angular acceleration experienced by the particle, and is called the "acceleration torque" here for later discussion. It is worth to compare Eq. (9) with the drag on the same sphere, $F$, when it is moving with an arbitrary time varying speed $U(t)$ along a straight path in a still fluid. According to Landau and Lifshitz. ${ }^{6}$

Downloaded 19 Dec 2008 to 140.112.113.225. Redistribution subject to AlP license or copyright; see http://apl.aip.org/apl/copyright.jsp

$$
\begin{aligned}
F= & 6 \pi \eta R U+\frac{2}{3} \rho \pi R^{3} \frac{d U}{d t} \\
& +6 \pi \eta R \frac{1}{\sqrt{\pi}} \int_{-\infty}^{t} \frac{d U}{d \tau} \frac{R}{\sqrt{\nu(t-\tau)}} d \tau .
\end{aligned}
$$

The terms on the right hand side of Eq. (10) are the quasisteady drag, the force associated with the added (virtual) mass, and the force associated with the acceleration history (known as the Basset force in literatures), respectively. By comparing the arbitrary rotation in Eq. (9) with the arbitrary linear translation in Eq. (10), both have a quasisteady term and a history term, but there is no counterpart for the added mass effect in the rotary motion. For the history effect, the expression for the associated viscous torque is more complicated and has two terms. The first history term has the same time dependence as that of the drag in Eq. (10), but the additional second term is more complicated and makes the integrand decay more rapidly at large time for rotary motion as observed by expanding the complementary error function in Eq. (9).

The validity of Eq. (9) may be checked by studying the long time response of the torque subject to rotary oscillation. By substituting $\Omega=\Omega_{\omega} e^{-i \omega t}$ when $t \geqslant 0$ and $\Omega=0$ when $t$ $<0$ into Eq. (9), and carrying out the integration with $t$ $\rightarrow \infty$, we found the same result as that in Eq. (2) as expected. The validity can also be checked for the case of a triangular forcing with 
TABLE II. Dimensionless time $(\bar{t})$ when $T_{a} / T_{\mathrm{qs}} \approx 0.01$ of the start-up problem for different $\overline{t_{0}}$.

\begin{tabular}{lllllc}
\hline \hline $\bar{t}_{0}$ & 0.01 & 0.1 & 1 & 10 & 100 \\
$\bar{t}$ & 3.65 & 3.70 & 4.17 & 10.7 & 30.0 \\
\hline \hline
\end{tabular}

$$
\begin{aligned}
\Omega & =0 \quad \text { when } t<0 \text { and } t>2 t_{0} \\
& =\Omega_{0} t / t_{0} \quad \text { when } 0 \leqslant t \leqslant t_{0} \\
& =\Omega_{0}\left(2-t / t_{0}\right) \quad \text { when } t_{0} \leqslant t \leqslant 2 t_{0},
\end{aligned}
$$

where $\Omega_{0}$ and $t_{0}$ are constants. By substituting Eq. (11) into Eq. (9), we found an expression for the dimensionless torque $T /\left(-8 \pi \eta R^{3} \Omega_{0}\right)$ in terms of the dimensionless times, $\bar{t}$ $=t /\left(R^{2} / \nu\right)$ and $\bar{t}_{0}=t_{0} /\left(R^{2} / \nu\right)$, normalized by the viscous time scale, $R^{2} / \nu$. On the other hand, Eq. (11) can also be expressed in terms of a Fourier series, and the torque for each Fourier component can be evaluated by using Eq. (2). Both approaches give consistent results (Table I).

The relative importance of the acceleration torque to the quasisteady torque can be studied via the start-up problem with

$$
\begin{aligned}
\Omega & =0 \quad \text { when } t<0 \\
& =\Omega_{0} t / t_{0} \quad \text { when } 0 \leqslant t \leqslant t_{0} \\
& =\Omega_{0} \quad \text { when } t>t_{0} .
\end{aligned}
$$

By substituting Eq. (12) into Eq. (9), we found

$$
T=T_{\mathrm{qs}}+T_{a},
$$

with the quasisteady torque

$$
\begin{aligned}
T_{\mathrm{qs}} & =-8 \pi \eta R^{3} \Omega_{0} \frac{t}{t_{0}}, \quad \text { for } 0 \leqslant t \leqslant t_{0} \\
& =-8 \pi \eta R^{3} \Omega_{0}, \quad \text { for } t>t_{0},
\end{aligned}
$$

and the acceleration torque

$$
\begin{aligned}
T_{a}= & \frac{T_{\mathrm{qs}}}{(3 \sqrt{\pi}) \bar{t}}\left\{2 \sqrt{\bar{t}}-\int_{0}^{\bar{t}} \sqrt{\pi} e^{x} \operatorname{erfc}(\sqrt{x}) d x\right\} \\
& \text { for } 0<t<t_{0} \\
= & \frac{T_{\mathrm{qs}}}{(3 \sqrt{\pi}) \bar{t}_{0}}\left\{2 \sqrt{\bar{t}}-2 \sqrt{\bar{t}-\bar{t}_{0}}\right. \\
& \left.-\int_{\bar{t}-\bar{t}_{0}}^{\bar{t}_{0}} \sqrt{\pi} e^{x} \operatorname{erfc}(\sqrt{x}) d x\right\} \text { for } t>t_{0} .
\end{aligned}
$$

Variations of $T_{a} / T_{\mathrm{qs}}$ with $\bar{t}$ for different values of $\bar{t}_{0}$ are calculated numerically and plotted in Fig. $1 . T_{a} / T_{\mathrm{qs}}$ decays quite rapidly as $\bar{t}$ increases, which implies that we can neglect the acceleration torque and employ the quasisteady torque to represent the resistive viscous torque when $t$ is sufficiently greater than the viscous time scale, $R^{2} / \nu$ (see Table II). For a given $\bar{t}_{0}, T_{a} / T_{\mathrm{qs}}$ decreases approximately with $1 / \sqrt{\bar{t}}$ at small $\bar{t}$, which is a consequence of the first term on the right hand side of Eq. (14c) dominating the second term at small time. As $\bar{t}$ increases, the second term rises and thus $T_{a} / T_{\mathrm{qs}}$ decays faster than $1 / \sqrt{\bar{t}}$. When $\bar{t}>\bar{t}_{0}$, the angular acceleration equals zero, and $T_{a} / T_{\mathrm{qs}}$ decays rapidly from the original trend. When $\bar{t}$ is sufficiently large, $T_{a} / T_{\mathrm{qs}} \propto(\bar{t})^{-3 / 2}$ approximately according to Fig. 1, which agrees with the theoretical analysis of Eq. (14d) by expanding the complementary error function at large $x$.

In general, the relative importance of the acceleration torque $T_{a}$ to the quasisteady torque $T_{\mathrm{qs}}$ can be studied through a scaling analysis of Eq. (9). As $T_{a}$ could be significant only at relatively small values of $(t-\tau) /\left(R^{2} / \nu\right)$, we may estimate $T_{a}$ using only its first term in the integral of Eq. (9) for studying if $T_{a}$ is negligible in comparing with $T_{\mathrm{qs}}$. Letting $t_{s}$ be the time scale of angular acceleration, we found $T_{a} / T_{\mathrm{qs}} \sim \sqrt{\left(R^{2} / \nu\right) / t_{s}}$ after a simple scaling analysis. If the rotation is driven by electrorotation or traveling wave dielectrophoresis, $t_{s}$ may be estimated as the relaxation time constant associated with the Maxwell-Wagner interfacial polarization, ${ }^{1}$ which is about $2.2 \times 10^{-3} \mathrm{~s}$ [using the data of Fig. 3.2(a) in Ref1]. For particle with $R \approx 5 \times 10^{-6} \mathrm{~m}$ in aqueous solution $\left(\nu \approx 10^{-6} \mathrm{~m}^{2} / \mathrm{s}\right), R^{2} / \nu \approx 2.5 \times 10^{-5} \mathrm{~s}$. It follows that $T_{a} / T_{\mathrm{qs}} \sim 0.1$, which implies that the acceleration torque could be negligible in comparing with the quasisteady torque. However, $T_{a}$ and $T_{\mathrm{qs}}$ are of the same order if the radius of the particle is increased by one order of magnitude, i.e., $R \approx 50 \mu \mathrm{m}$.

As the problem is linear, the scalar equation in Eq. (9) can be extended to its vector form by replacing $T$ and $\Omega$ with the torque vector $\mathbf{T}$ and the angular velocity $\boldsymbol{\Omega}$, respectively. In case the particle is rotating in a velocity field, $\mathbf{V}(x, y, z, t)$, instead of in a still fluid, the torque can be expressed as

$$
\begin{aligned}
\mathbf{T}(t)= & -8 \pi \eta R^{3}\left(\boldsymbol{\Omega}-\frac{1}{2} \nabla \times \mathbf{V}\right) \\
& -\frac{8 \pi \eta R^{3}}{3 \sqrt{\pi}} \int_{-\infty}^{t}\left[\frac{R}{\sqrt{\nu(t-\tau)}}\right. \\
& \left.-\sqrt{\pi} \exp \left(\frac{\nu(t-\tau)}{R^{2}}\right) \operatorname{erfc}\left(\frac{\sqrt{\nu(t-\tau)}}{R}\right)\right] \\
& \times \frac{d(\boldsymbol{\Omega}-(1 / 2) \nabla \times \mathbf{V})}{d \tau} d \tau,
\end{aligned}
$$

by using Faxen's law in a way similar to that by Happel and Brenner $^{7}$ (p. 68, see also Ref. 5). Here the term (1/2) $\nabla$ $\times \mathbf{V}$ is evaluated at the instantaneous location of the center of the sphere.

The work is supported partially by the National Science Council of Taiwan, Republic of China through Grant No. NSC 94-2120-M-002-014.

${ }^{1}$ T. B. Jones, Electromechanics of Particles (Cambridge University Press, Cambridge, 1995).

${ }^{2}$ M. P. Hughes, Nanotechnology 11, 124 (2000).

${ }^{3}$ Y. Huang, X.-B. Wang, J. A. Tame, and R. Pethig, J. Phys. D 26, 1528 (1993).

${ }^{4}$ H. Lamb, Hydrodynamics (Dover, New York, 1945).

${ }^{5}$ M. R. Maxey and J. Riley, Phys. Fluids 26, 883 (1983).

${ }^{6}$ L. E. Landau and E. M. Lifshitz, Fluid Mechanics (Pergamon, New York, 1959).

${ }^{7}$ J. Happel and H. Brenner, Low Reynolds Number Hydrodynamics (Nijhoff, Boston, 1986). 